\title{
Man-in-the-Middle Secure Authentication Schemes from LPN and Weak PRFs
}

\author{
Vadim Lyubashevsky ${ }^{1}$ and Daniel Masny ${ }^{2, \star}$ \\ 1 INRIA / École Normale Supérieure, Paris \\ lyubash@di.ens.fr \\ 2 Ruhr-Universitat Bochum \\ daniel.masny@ruhr-uni-bochum.de
}

\begin{abstract}
We show how to construct, from any weak pseudorandom function, a 3-round symmetric-key authentication protocol that is secure against man-in-the-middle attacks. The construction is very efficient, requiring both the secret key and communication size to be only $3 n$ bits long and involving only one call to the weak-PRF. Our techniques also extend to certain classes of randomized weak-PRFs, chiefly among which are those based on the classical LPN problem and its more efficient variants such as Toeplitz-LPN and Ring-LPN. Building an efficient manin-the-middle secure authentication scheme from any weak-PRF resolves a problem left open by Dodis et al. (Eurocrypt 2012), while building a man-in-the-middle secure scheme based on any variant of the LPN problem solves the main open question in a long line of research aimed at constructing a practical light-weight authentication scheme based on learning problems, which began with the work of Hopper and Blum (Asiacrypt 2001).
\end{abstract}

\section{Introduction}

The need for light-weight cryptography is increasing rapidly due to the growing deployment of low-cost devices, such as smart cards and RFID tags, in the real world. One of the most common cryptographic protocols required on these devices is a symmetric key authentication protocol in which the prover (usually referred to as the Tag) authenticates his identity to the verifier (usually referred to as the Reader). The most direct way in which this protocol can be constructed is by using a pseudorandom function $f$ (e.g. AES) for which the Tag and the Reader share a common key. Then the authentication protocol simply consists of the Reader sending a challenge $c$ to which the Tag replies with $f(c)$, and the Reader verifies that the received evaluation of $c$ is indeed correct. The main problem with this approach is that the pseudorandom function, whether it is a "provably-secure" one based on some mathematical assumption or an "ad-hoc" block cipher like AES, is usually quite costly for light-weight devices. For this reason, researchers have worked on designing block ciphers specifically

* Part of this work was done while visiting École Normale Supérieure, Paris.

R. Canetti and J.A. Garay (Eds.): CRYPTO 2013, Part II, LNCS 8043, pp. 308-325, 2013.

(C) International Association for Cryptologic Research 2013 
for low-cost devices (e.g. 19/4]). A different approach for solving this problem is constructing authentication schemes from building blocks that have weaker security properties than block ciphers or pseudorandom functions. We pursue this latter avenue of research in the present work.

\subsection{Authentication from LPN}

The Learning Parity with Noise (LPN) problem was initially shown to have cryptographic applications by Goldreich et al. and Blum et al. in [113], and then used as a basis for authentication schemes by Hopper and Blum in their HB scheme [14]. In this latter paper, a simple LPN-based authentication scheme was proposed that was secure in the passive attack model. Later work by Juels and Weis [15, and also by Katz and Shin [16, modified this protocol (the result was called $\mathrm{HB}^{+}$) to be secure against active adversaries. Nevertheless, even these schemes had a serious security shortcoming. If the adversary were allowed to modify the communication between the Tag and the Reader and observe the response of the reader to verification queries, then, as shown by Gilbert et al. [7, there exists a very simple attack that can recover the secret key in polynomial time. Because such a man-in-the-middle attack can be mounted with relatively small effort, schemes that fall to it cannot be considered secure enough for realworld applications that require some decent level of security. It was thus a major open problem to construct an efficient LPN-based authentication scheme that remains secure against man-in-the-middle attacks.

A notable advance was made by Gilbert et al. [9] who proposed a scheme (termed $\mathrm{HB}^{\#}$ ) that was able to resist the attack from [7] and was shown to be secure against restricted man-in-the-middle adversaries. A second contribution of this work was to offer a solution to another problematic feature of previous LPN-based protocols. All protocols that are based on LPN require either the key size or the communication complexity to be square in the security parameter. Thus either the key size or the communication complexity would have to be on the order of hundreds of thousands of bits. Since the main motivation for LPN-based protocols is low-cost hardware, this is clearly unacceptable. To this end, 9] proposed a protocol based on a related assumption, called ToeplitzLPN (see Section 2.2 for definitions), where the communication complexity was small and the secret key had some structured form which allowed for compact representations. While there has been no known weakness caused by using the Toeplitz-LPN assumption, it did turn out that the restricted man-in-the-middle model introduced in [9] was not sufficient to prevent all practical attacks, and one such attack was shown by Ouafi et al. 24].

There have been many other proposals, some without security proofs, others with claimed proofs that attempted to solve this problem, but all of these methods were ultimately shown to be flawed (see [8] for a small overview). A breakthrough finally came in a series of recent papers by Kiltz et al. [18] and Dodis et al. [6] who constructed relatively-efficient MACs based on the hardness of the LPN problem. Because MACs immediately give rise to man-inthe-middle secure authentication schemes, their work also resolved the problem 
of building such schemes from the LPN problem. This LPN MAC, however, suffered from the same drawback as other LPN-based schemes - the key size was prohibitively large. Thus in order to be useful in practice, the proof techniques would have to be adapted to work with more compact LPN-related assumptions, such as Toeplitz-LPN. But the constructions of [18] and 6] made use of certain algebraic structure of the LPN-problem, and the proofs turn out to be incompatible with other previously-considered versions of LPN.

\subsection{Authentication from Weak-PRFs}

Weak pseudo-random functions are keyed functions whose outputs on random inputs are indistinguishable from uniform. Weak PRFs are considered to be much "weaker" primitives than PRFs, and in particular, it is not known how to transform a weak-PRF into a PRF except by using tree techniques similar to the classical GGM construction [10. Additionally, it also appears to be much easier to build secure weak-PRFs than PRFs. For example, the function $f_{a}(x)=x^{a} \bmod p$ is a weak-PRF based on the DDH assumption, whereas the construction of a PRF based on $\mathrm{DDH}$ is much less efficient [22], requiring $n$ multiplications in addition to the exponentiation in the weak-PRF. Similarly, the recent construction of lattice-based PRFs 1 first builds a relatively efficient weak-PRF (which is just $f_{A}(x)=\operatorname{Round}\left(A x \bmod p\right.$ ), where $A \in \mathbb{Z}_{p}^{m \times n}, x \in \mathbb{Z}^{n}$ with $\|x\|$ small, and the $\operatorname{Round}(\cdot)$ function drops a super-logarithmic number of least-significant bits) and then converts it to a full PRF using techniques similar to 22 23. The resulting lattice-based PRF is both less efficient and requires a stronger computational assumption than the underlying weak-PRF.

Due to efficiency advantages and lower security requirements, there has been some research on constructions of cryptographic primitives such as symmetric encryption and stream ciphers built directly from weak-PRFs (e.g. [5 2125]). The work along this theme that is most related to ours is the aforementioned one of Dodis et al. [6], where it is shown how to build a 3-round authentication scheme secure against active attacks from any weak-PRF. As we mentioned earlier, the active security model, where the adversary is not allowed to send any verification queries to the Reader, is not considered strong enough for realworld applications. And so the problem of constructing man-in-the-middle secure authentication schemes from arbitrary weak-PRFs remained open.

\subsection{Our Results}

Our first result is a construction, from any weak pseudorandom function, of a 3 -round symmetric-key authentication protocol that is secure against man-inthe-middle attacks. Our scheme has the exact same communication complexity as the actively-secure scheme of [6], and only has one extra key element. To be more precise, the secret keys in our scheme consist of the key of the weak-PRF plus the description of a pairwise-independent hash function, which requires an additional two elements, whose size is the output length of the weak-PRF. So if 
we assume that both the domain and range of the weak-PRF is $n$ bits, then the total key size is $3 n$.

We then extend our construction of a weak-PRF scheme to randomized weak-PRFs. Randomized weak-PRFs are keyed functions that become computationally indistinguishable from uniform when their outputs are perturbed by some low-entropy noise. Noisy learning problems such as LPN and LWE 26] can be equivalently viewed as problems of distinguishing the outputs of a randomized weak-PRF from the uniform distribution. To get a man-in-themiddle secure authentication scheme from a randomized weak-PRF, we require just one more secret key element than our weak-PRF based scheme.

Our constructions, and to some extent their security proofs as well, turn out to be surprisingly simple. The main insight is that one should embed the $n$-bit output of the (randomized) weak-PRF into a finite field of size $2^{n}$. Then, in addition to the secret keys associated to the function, we also create secret keys in the field which end up being masked by the presumed indistinguishability from uniform of the (randomized) weak-PRF. We then show how the interplay in the field between the weak-PRF and the additional secret keys results in protocols that have the desired man-in-the-middle security.

We prove security of our schemes in the sequential man-in-the-middle model, in which the adversary simultaneously interacts with one copy of the Tag and Reader (see Figure 1). The schemes remain secure even if the adversary has access to multiple readers (this is shown in the full version of the work), whereas concurrent access to multiple tags may result in a vulnerability 1 In the stronger notion of concurrent man-in-the-middle security the adversary is allowed to simultaneously communicate with multiple copies of the Tag and Reader. While the concurrent model is theoretically stronger, we do not believe that it is practically relevant to the low-cost device setting considered in this paper. In particular, it is unlikely that a low-cost Tag would have the need (or ability) to simultaneously participate in more than one authentication session. Furthermore, it also seems imprudent that in an ecosystem where one wants to have relatively strong security, secret keys would be shared among the Tags. Still, constructing an efficient authentication scheme from generic weak-PRFs that is secure in the concurrent man-in-the-middle model is an interesting open problem.2

\subsection{Comparison to Other Works}

Table 1 compares the results obtained in this paper with those of previous works. Compared to the protocols that only achieve active security, our scheme achieves the much stronger man-in-the-middle security at a fairly small cost. In the case of protocols based on a generic weak-PRF, we extend the security to the man-in-the middle model at the cost of only one extra secret key element and one extra field multiplication. We get similar results when comparing our protocol with actively-secure LPN-based ones.

\footnotetext{
${ }^{1}$ In the full version of the paper, we show that for certain instantiations of a randomized weak-PRF there indeed exists a concurrent man-in-the-middle attack.

${ }^{2}$ Our current scheme is still secure in the concurrent model against active attacks.
} 
Table 1. Authentication Protocols Based on Weak-PRFs and the LPNrelated Assumptions. Listed is the amount of authentication rounds \# $r$, the security properties achieved by the protocol and its complexity (with lower order terms dropped) according to the key size and the communication. Let $\epsilon$ be the advantage in breaking the assumption, then the term depending on $\epsilon$ is proven to be the best possible advantage of breaking the protocol in the given model. $Q$ is the amount of tag and verification queries whereas $q_{v}$ is defined as the amount of verification queries, which is $q_{v}=1$ in the active model. $n$ parameterizes the hardness of the assumption and $\lambda$ is the statistical security parameter. 6] gives an alternate construction of $\mathrm{MAC}_{1}$ and $\mathrm{MAC}_{2}$ with better computational complexity, but the rest of the properties are basically the same.

\begin{tabular}{|c|c|c|c|c|c|c|}
\hline \multirow{2}{*}{ Protocol } & \multirow{2}{*}{$\# r$} & \multicolumn{3}{|c|}{ Security } & \multicolumn{2}{|c|}{ Complexity } \\
\hline & & assumption & active & MIM & key size & com. \\
\hline weak-PRF [6] & 3 & weak-PRF & $\sqrt{\epsilon}$ & $?$ & $2 n$ & $3 n$ \\
\hline weak-PRF [this work] & 3 & weak-PRF & \multicolumn{2}{|c|}{$q_{v} \cdot \sqrt{\epsilon}$} & $3 n$ & $3 n$ \\
\hline $\left.\mathrm{HB}^{+}[15] 16\right]$ & 3 & $\overline{\mathrm{LPN}_{n, \tau}}$ & $\overline{|c|}$ & $\overline{\mathrm{X}[7]}$ & $2 n$ & $\overline{2 n^{2}}$ \\
\hline Random-HB $^{\#}$ [9] & 3 & $\mathrm{LPN}_{n, \tau}$ & $\sqrt{\epsilon}$ & $\mathrm{X}[24$ & $2 n^{2}$ & $3 n$ \\
\hline $\mathrm{HB}^{\#}$ [9] & 3 & Toeplitz-LPN $_{n, \tau}$ & $\sqrt{\epsilon}$ & $\mathrm{X}[24$ & $4 n$ & $3 n$ \\
\hline $\mathrm{MAC}_{1}[18$ & 2 & $\mathrm{LPN}_{n, \tau}$ & $\epsilon$ & $2^{\lambda} \cdot \epsilon$ & $2 n^{2}$ & $4 n$ \\
\hline $\mathrm{MAC}_{2}[18$ & 2 & $\mathrm{LPN}_{n, \tau}$ & $\epsilon$ & $Q \cdot \epsilon$ & $\lambda n^{2}$ & $4 n$ \\
\hline Lapin 13 & 2 & Ring-LPN $_{n, \tau}$ & $\epsilon$ & $?$ & $2 n$ & $3 n$ \\
\hline $\mathrm{MAC}_{1}+$ Lapin & 2 & Ring-LPN ${ }_{n, \tau}$ & $\epsilon$ & $2^{\lambda} \cdot \epsilon$ & $6 n+2 \lambda$ & $4 n$ \\
\hline \multirow{3}{*}{ LPN-based [this work] } & \multirow{3}{*}{3} & $\mathrm{LPN}_{n, \tau}$ & \multirow{3}{*}{\multicolumn{2}{|c|}{$q_{v} \cdot \sqrt{\epsilon}$}} & $n^{2}$ & \multirow{3}{*}{$3 n$} \\
\hline & & Toeplitz-LPN $_{n, \tau}$ & & & $5 n$ & \\
\hline & & Ring-LPN $_{n, \tau}$ & & & $4 n$ & \\
\hline
\end{tabular}

It is also interesting to compare our LPN protocol to the MAC constructions in 18. There are three advantages to the MAC constructions - they are only two rounds, they have slightly tighter reductions to LPN, and they are secure in the concurrent man-in-the-middle model, whereas our scheme is secure in the sequential man-in-the-middle model. The advantages of our construction are that the key sizes and the communication complexities are smaller.

The above-listed differences between our LPN scheme and the MAC schemes are, in our opinion, fairly minor with several pluses and minuses on both sides. In practice, it makes almost no difference whether the authentication scheme is 2 or 3 rounds since the Tag is the one who starts the protocol - thus a 2round protocol essentially becomes a 3-round one. And while security tightness is certainly a desirable property, it is very unclear what effects it has in practice. Similar public key authentication schemes, such as GQ [12] and Schnorr [27, have been studied for a long time, yet do not exhibit any weaknesses due to their non-tight reductions.

The major advantage of our construction is that it is generic and can be instantiated with virtually any version of the LPN function or a randomized weak-PRF satisfying a few mild properties (see Section 2.1). For example, our construction allows for authentication schemes based on the fairly well-studied 
Toeplitz-LPN assumption, which seems to provide a very good compromise between security and computational efficiency. The constructions of [18] and [6], on the other hand, can only construct MACs from functions with very "algebraic" properties.

The recent work of Heyse et al. 13. proposed a new LPN-type assumption, called Ring-LPN, to enable efficient constructions that are compatible with the MAC transformation in [18. The assumption is relatively new, and its unclear at this point whether it has the same hardness as the more well-studied LPN and Toeplitz-LPN assumptions. Still, even if the Ring-LPN problem is hard, our LPN protocol can also be instantiated based on this assumption and is more efficient than the resulting MAC transformation.

\section{Preliminaries and Notation}

\subsection{Function Families and Their Properties}

In this section we define the important classes of functions that will appear in the paper. As mentioned earlier, we will be considering embeddings of function outputs into a finite field. The embedding can be arbitrary, and the simplest one is to simply think of a function output string $s \in\{0,1\}^{n}$ as a polynomial in a finite field $\mathbb{F}=\left(\mathbb{Z}_{2}^{n},+, \times\right)$ for appropriately defined addition and multiplication operations. Thus, without loss of generality, we will assume that all our functions output elements to some finite field $\mathbb{F}$.

Definition 2.1. A function family $\mathcal{H}: \mathbb{D} \rightarrow \mathbb{F}$ is called pairwise-independent if for all $x_{1} \neq x_{2} \in \mathbb{D}, y_{1}, y_{2} \in \mathbb{F}$,

$$
\underset{h \stackrel{\oplus}{\leftarrow} \mathcal{H}}{\operatorname{Pr}}\left[h\left(x_{1}\right)=y_{1} \wedge h\left(x_{2}\right)=y_{2}\right]=1 /|\mathbb{F}|^{2} .
$$

Definition 2.2. A function family $\mathcal{F}: \mathbb{D} \rightarrow \mathbb{F}$ is said to be a weak-PRF family if for any polynomial-sized $k$, randomly-chosen $f \in \mathcal{F}$, and randomly-chosen $r_{1}, \ldots, r_{k} \in \mathbb{D}$, the distribution of $\left(r_{1}, f\left(r_{1}\right)\right), \ldots,\left(r_{k}, f\left(r_{k}\right)\right)$ is computationally indistinguishable from the uniform distribution over $(\mathbb{D}, \mathbb{F})^{k}$.

Even if $\left(r_{1}, f\left(r_{1}\right)\right), \ldots,\left(r_{k}, f\left(r_{k}\right)\right)$ can be distinguished from the uniform distribution over $(\mathbb{D}, \mathbb{F})^{k}$, it's possible that the sequence can become indistinguishable if the outputs $f\left(r_{i}\right)$ were perturbed by some noise. Such function families are called randomized weak-PRFs. The noise perturbation can be anything, but in this paper we will only consider noise distributions with an eye towards LPN applications. In particular, both the noise and the output of $f\left(r_{i}\right)$ are group elements, and the perturbation consists of adding the two together. This is still consistent with our requirement of being able to embed the output of all functions into a finite field $\mathbb{F}$ since the group needed for LPN can simply be the underlying additive group of $\mathbb{F}$ (see Section 2.2 ). 
Definition 2.3. For a function $f(\cdot): \mathbb{D} \rightarrow \mathbb{F}$ and a distribution $\chi$ over $\mathbb{F}$, we will write $f^{\chi}(r)$ to mean a randomized function that generates an element $e \in \mathbb{F}$ according to the distribution $\chi$ and outputs $f(r)+e$. A function family $\mathcal{F}: \mathbb{D} \rightarrow \mathbb{F}$ is said to be a randomized weak-PRF family with noise $\chi$ if for any polynomialsized $k$, randomly-chosen $f \in \mathcal{F}$, and randomly-chosen $r_{1}, \ldots, r_{k} \in \mathbb{D}$, the distribution of $\left(r_{1}, f^{\chi}\left(r_{1}\right)\right), \ldots,\left(r_{k}, f^{\chi}\left(r_{k}\right)\right)$ is computationally indistinguishable from the uniform distribution over $(\mathbb{D}, \mathbb{F})^{k}$.

In order for randomized weak-PRFs to be useful for cryptographic constructions, the range $\mathbb{F}$ and the error distribution should have certain characteristics. For example, the weak-PRFs would be of very little use if the error distribution $\chi$ was just the uniform distribution over $\mathbb{F}$. In this paper we will assume that the additive group of the field $\mathbb{F}$ and the error distribution $\chi$ satisfy the following three properties:

1. There exists a weight function $\|\cdot\|: \mathbb{F} \rightarrow \mathbb{R}^{+}$such that the additive group that underlies the field $\mathbb{F}$ satisfies the triangle inequality - that is for all $a, b \in \mathbb{F},\|a \pm b\| \leq\|a\|+\|b\|$. Additionally, $\|a\|=0$ if and only if $a=0$.

2. There exists a positive real $\tau^{\prime} \in \mathbb{R}$ such that $\operatorname{Pr}_{e \sim \chi}\left[\|e\| \leq \tau^{\prime}\right]=1-n^{-\omega(1)} 3$

3. For a positive real $\alpha$, let $\beta(\alpha)=\{z \in \mathbb{F}:\|z\| \leq \alpha\}$. We will assume that $\left|\beta\left(2 \tau^{\prime}\right)\right| /|\mathbb{F}|=n^{-\omega(1)}$.

The first property essentially makes sure that the randomness in the randomized weak-PRF behaves "nicely" via the triangular inequality 4 The second property determines the completeness of our protocol. Additionally, because of the way our security proof works, the completeness of the protocol also plays a role in the soundness of the protocol 5 Thus this value should be very close to 1 . The third property determines the soundness of the protocol. Intuitively, it is related to the probability that an adversary can randomly guess a response and be accepted by the verifier.

Due to their similarity, we will be presenting our authentication scheme and its proof based on weak-PRFs together with the ones based on randomized weak-PRFs. Since a weak-PRF is just a randomized weak-PRF whose error distribution $\chi$ has its support entirely on 0, it's easy to see that it can trivially be made to satisfy the above three properties. We can define the weight function as $\|x\|=1$ for all $x \neq 0$ and set $\tau^{\prime}=0$. Thus for weak-PRFs we have $\operatorname{Pr}_{e \sim \chi}[\|e\| \leq$ $\left.\tau^{\prime}\right]=1$ (and so the protocol will have perfect completeness) and $\left|\beta\left(2 \tau^{\prime}\right)\right| /|\mathbb{F}|=$ $|\{0\}| /|\mathbb{F}|=1 /|\mathbb{F}|$.

${ }^{3}$ More formally, $\tau^{\prime}$ is a function of $n, \tau^{\prime}(n)$, but we will omit the $n$ throughout the paper.

4 Even though we are using the standard notation for "norm", the weight function $\|\cdot\|$ is not quite a norm because it's not true that for all integers $\alpha, \alpha\|a\|=\|\alpha a\|$ (since we are working over a finite field).

5 This seems to be a common feature of protocols that have man-in-the-middle security because the simulator replies to the adversary under the assumption that properlyformed responses by the Tag are accepted by the Reader. Even though it is not stated in [186], the soundness of their protocols also depends on their completeness in exactly the same way as in this work. 


\subsection{Randomized Weak-PRFs from the LPN Problem and Its Variants}

The classical decisional $\mathrm{LPN}_{n, \tau}$ assumption states that the uniform distribution over $\mathbb{Z}_{2}^{n} \times \mathbb{Z}_{2}$ is computationally-indistinguishable from the following distribution: for a fixed randomly-chosen vector $s \in \mathbb{Z}_{2}^{n}$, output $(r, r \cdot s+e)$ where $r$ is chosen uniformly random from $\mathbb{Z}_{2}^{n}$ and $e$ is a Bernoulli random variable that is 1 with probability $\tau$. By the hybrid argument, it is easy to see that if the fixed secret is now a matrix $S \in \mathbb{Z}_{2}^{m \times n}$ then the distribution $(r, S r+e)$, where $r$ is chosen as before and $e$ is a vector each of whose coefficients is 1 with probability $\tau$, is also computationally-indistinguishable from the uniform distribution over $\mathbb{Z}_{2}^{n} \times$ $\mathbb{Z}_{2}^{m}$ (with a loss of a factor $m$ in the reduction). We now formulate this latter statement in terms of the randomized weak-PRF notation from the previous subsection.

Let $B e r_{\tau}^{m}$ be a distribution over $\mathbb{Z}_{2}^{m}$ where every coordinate is independently chosen to be 1 with probability $\tau$ and 0 with probability $1-\tau$.

Definition 2.4 (LPN). Let $\mathcal{F}: \mathbb{Z}_{2}^{n} \rightarrow \mathbb{Z}_{2}^{m}$ be a function family indexed by matrices $S \in \mathbb{Z}_{2}^{m \times n}$. For a function $f_{S} \in \mathcal{F}$ and a vector $r \in \mathbb{Z}_{2}^{n}$, define $f_{S}(r):=$ $S r$. Then the $L P N_{n, \tau}$ assumption implies that $\mathcal{F}$ is a randomized weak-PRF family with noise $\mathrm{Ber}_{\tau}^{m}$.

In the above definition, the domain $\mathbb{D}$ of $\mathcal{F}$ is $\mathbb{Z}_{2}^{n}$. Because we insisted in Definition 2.3 that the range of the function family $\mathcal{F}$ be a finite field (this will be used in our protocol) and the LPN problem only requires an additive group structure, we have some freedom as to how to define this field. The LPN assumption requires the range to have the group structure $\left(\mathbb{Z}_{2}^{m},+\right)$, thus $\mathbb{F}$ can be any finite field that has $\left(\mathbb{Z}_{2}^{m},+\right)$ as its underlying additive group. The most natural definition is $\mathbb{F}=\mathbb{Z}_{2}[x] /(g(x))$ where $g(x)$ is a polynomial of degree $m$ that is irreducible over $\mathbb{Z}_{2}$, and addition and multiplication are just standard polynomial addition and multiplications modulo 2 and $g(x)$. Thus addition in $(\mathbb{F},+, \times)$ exactly corresponds to addition in $\left(\mathbb{Z}_{2}^{m},+\right)$.

The randomized weak-PRF based on LPN can also quite naturally be made to satisfy the three properties after Definition 2.3 . The weight function $\|\cdot\|$ can be defined to be the Hamming weight. That is, for any element $a \in \mathbb{Z}_{2}^{m},\|a\|$ is the number of 1's in $a$. With this definition of the weight function, one can compute, via the Chernoff bound, a $\tau^{\prime}$ such that any element $e$ chosen according to $B e r_{\tau}^{m}$ satisfies $\|e\| \leq \tau^{\prime}$ with overwhelming probability. To satisfy the third property, we would need that $\left|\beta\left(2 \tau^{\prime}\right)\right| /|\mathbb{F}|=n^{-\omega(1)}$, which is equivalent to the condition that $\left(\sum_{i=0}^{\left\lfloor 2 \tau^{\prime}\right\rfloor}\left(\begin{array}{c}m \\ i\end{array}\right)\right) / 2^{m}=n^{-\omega(1)}$. The above conditions are identical to those in other authentication protocols, such as [16]17/9, and so the LPN parameters needed to make those schemes secure, also carry over to ours.

Because the LPN problem yields rather inefficient schemes, Gilbert et al. 9] proposed protocols based on the hardness of the Toeplitz-LPN problem, which is just like the LPN problem except that the secret matrix $S$ is a Toeplitz matrix. 


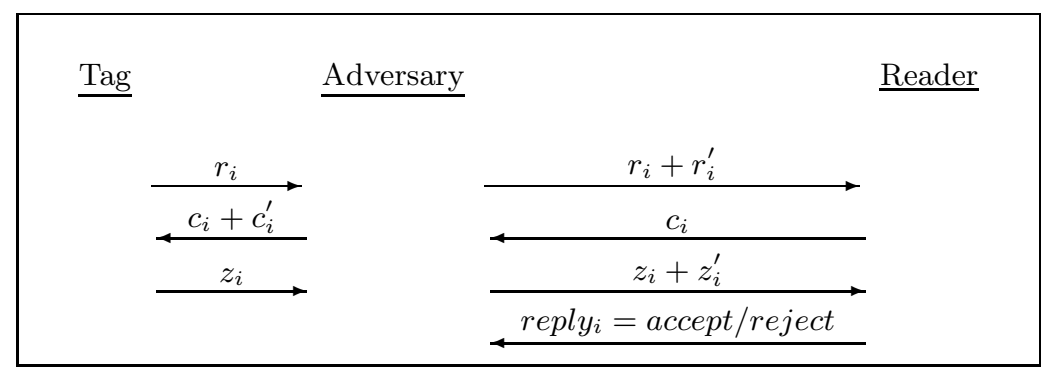

Fig. 1. Man-in-the-Middle Attack Model

Definition 2.5 (Toeplitz-LPN). Let $\mathcal{F}: \mathbb{Z}_{2}^{n} \rightarrow \mathbb{Z}_{2}^{m}$ be a function family indexed by Toeplitz matrices $S \in \mathbb{Z}_{2}^{m \times n}$. For a function $f_{S} \in \mathcal{F}$ and a vector $r \in \mathbb{Z}_{2}^{n}$, define $f_{S}(r):=S r$. Then the Toeplitz-LPN $N_{n, \tau}$ assumption implies that $\mathcal{F}$ is a randomized weak-PRF family with noise $B_{e} r_{\tau}^{m}$.

Heyse et al. 13] recently introduced the Ring-LPN problem, which also results in more efficient protocols. While the Ring-LPN problem has not been well-studied, it does have some resemblance to the better-studied Ring-LWE problem [20] in lattice cryptography, and so there are some reasons to believe that it might be secure.

Definition 2.6 (Ring-LPN). Let $g(x)$ be a polynomial of degree $n$ in $\mathbb{Z}_{2}[x]$ irreducible over $\mathbb{Z}_{2}$ and define the field $\mathbb{F}$ to be $\mathbb{F}=\mathbb{Z}_{2}[x] /((g(x))$. Let $\mathcal{F}: \mathbb{F} \rightarrow \mathbb{F}$ be a function family indexed by polynomials $s \in \mathbb{F}$. For a function $f_{s} \in \mathcal{F}$ and a polynomial $r \in \mathbb{F}$, define $f_{s}(r):=$ sr. Then the Ring-LPN $N_{n, \tau}$ assumption implies that $\mathcal{F}$ is a randomized weak-PRF family with noise $\mathrm{Ber}_{\tau}^{n}$.

\subsection{Security Models}

All authentication schemes are protocols in which the Tag and the Reader possess some secret key $s k$ and then perform an interaction in which the Tag must convince the Reader of his identity. The difference in the security models depends on the strength that we give the adversary. The three most natural security models are passive, active, and man-in-the-middle. All three models consist of two stages. In the first stage, depending on the model, the Adversary is allowed to have some interaction with the Tag and the Reader. In the second stage, in all three models, he loses the interaction with the Tag and must interact with the Reader in hopes of getting the latter to accept the interaction.

Man-in-the-Middle Adversary. The strongest type of Adversary is one who in the first stage is able to simultaneously interact with the Tag and the Reader and make verification queries to the Reader. In the second stage, the Adversary loses access to the Tag, and interacts with the Reader hoping to make the latter accept. 
In this paper, the protocols we will be constructing will be sigma protocols (i.e. have three rounds usually referred to as commit, challenge, and response) and will use a model that is simpler to describe and is at least as secure as the man-in-the-middle one. We now describe the security game and the Adversary's condition for winning it:

Setup: Generate a secret key and give it to the Tag $\mathcal{T}$ and the Reader $\mathcal{R}$.

Attack: Invoke the Adversary $\mathcal{A}$ who has access to $\mathcal{T}$ and $\mathcal{R}$ and let him interact with them $t$ times. Each of the interactions is as follows (see Figure 1):

$\mathcal{A}$ receives a commitment $r_{i}$ from $\mathcal{T}$ and sends a commitment $r_{i}+r_{i}^{\prime}$ to $\mathcal{R}$. $\mathcal{R}$ responds with a challenge $c_{i}$ and $\mathcal{A}$ sends a challenge $c_{i}+c_{i}^{\prime}$ to $\mathcal{T}$. $\mathcal{T}$ answers with a valid response $z_{i}$. $\mathcal{A}$ can now send his response $z_{i}+z_{i}^{\prime}$ for verification to $\mathcal{R}$. $\mathcal{R}$ answers with accept, if $\left(r_{i}+r_{i}^{\prime}, c_{i}^{\prime}, z_{i}+z_{i}^{\prime}\right)$ is valid according to the verification function, otherwise he answers with reject.

Winning Condition: We say that the Adversary $\mathcal{A}$ wins the game if at some point he makes a query to $\mathcal{R}$ such that $\left(r_{i}^{\prime}, c_{i}^{\prime}, z_{i}^{\prime}\right) \neq(0,0,0)$ and the Reader $\mathcal{R}$ sends reply $=$ accept.

Notice that if there is an Adversary who can win the two stage Man-in-theMiddle game (i.e. where he loses access to the Tag in the second stage and must get the reader to accept), then he can also win the game described above since he can simply ignore the messages sent by the Tag in the second stage. Thus security in the model that we will be using in this paper implies security in the "more natural" two stage model.

\section{Construction Based on a (Randomized) Weak-PRF}

In this section we present our main construction, an authentication protocol secure against man-in-the-middle attacks from any weak-PRF or a randomized weak-PRF that satisfies the three properties stated after Definition 2.3. The protocol based on a weak-PRF is very similar to the one based on a randomized weak-PRF, and so we present them together in Figure 2. The security proofs are also very similar, and we also present them together in the next section.

The underlying building blocks of the protocol in Figure 2 are a pairwiseindependent function family $\mathcal{H}$ and a family $\mathcal{F}$ of randomized weak-PRFs with noise $\chi$. If $\mathcal{F}$ is a family of standard (non-randomized) weak-PRFs, then it's the same as a randomized weak-PRF with noise $\chi$, where $\chi$ has all of its support on 0 - thus for all $f \in \mathcal{F}, f^{\chi}(\cdot)=f(\cdot)$. The secret keys of the authentication scheme are randomly chosen $f \in \mathcal{F}, h \in \mathcal{H}$, and $s \in \mathbb{F}$. In the case that $\mathcal{F}$ is a standard weak-PRF family, we do not need the extra key $s$, and in the protocol we can assume that $s=1$. In the case that $\mathcal{F}$ is a randomized weak-PRF family, we assume that it satisfies the three properties after Definition 2.3. Thus there is an associated weight function $\|\cdot\|$ and a value $\tau^{\prime}$ such that the error $e$ chosen from $\chi$ satisfies $\|e\| \leq \tau^{\prime}$ with overwhelming probability. 


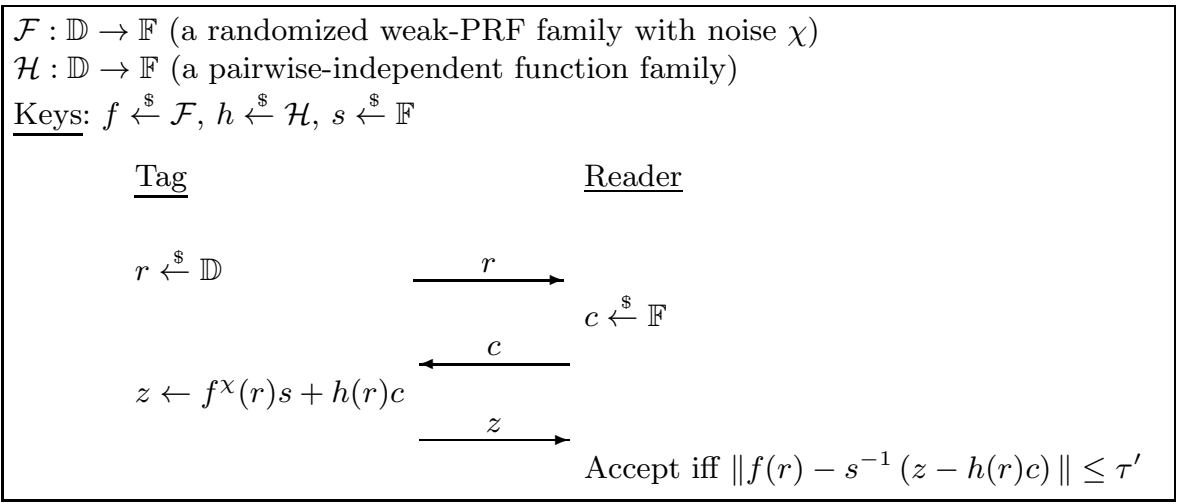

Fig. 2. Authentication Protocol Based on a (randomized) weak-PRF. If the weak-PRF is not randomized, (i.e. the support of the distribution $\chi$ is 0 and $\tau^{\prime}=0$ ), then we can set $s=1$. In this case, the condition $\left\|f(r)-s^{-1}(z-h(r) c)\right\| \leq \tau^{\prime}$ simplifies to $f(r)=z-h(r) c$.

In the first step of the protocol, the Tag picks a random element $r \in \mathbb{D}$ and sends it to the Reader. The reader chooses a random $c \in \mathbb{F}$ and sends it to the Tag. In its turn, the Tag evaluates $f^{\chi}(r)$ and $h(r)$, and sends $z=f^{\chi}(r) s+h(r) c$ back to the Reader, where all addition and multiplication operations take place in the field $\mathbb{F}$. In the case that $\mathcal{F}$ is a standard weak-PRF family, the response of the Tag is simply $z=f(r)+h(r) c$. The Reader accepts the Tag if $\| f(r)-$ $s^{-1}(z-h(r) c) \| \leq \tau^{\prime}$. In case of a standard weak-PRF family without noise, this condition is equivalent to $f(r)=z-h(r) c$.

Example Instantiation. We now give an example instantiation of the protocol using the $\mathrm{LPN}_{n, \tau}$ assumption from Definition [2.4. The noise distribution $\chi$ is $B e r_{\tau}^{m}$ and to choose the secret key $f$, a random $S \in \mathbb{Z}_{2}^{n \times m}$ is picked and $f^{\chi}(r):=$ $S r+e$ where $e \sim B e r_{\tau}^{m}$. Thus $f$ maps the domain $\mathbb{Z}_{2}^{n}$ to $\mathbb{Z}_{2}^{m}$. As in the discussion following Definition 2.4 , the field $\mathbb{F}$ is defined to be $\mathbb{Z}_{2}[x] /(g(x))$ where $g(x)$ is any irreducible polynomial of degree $m$. The simplest definition of a pairwise independent function family that maps $\mathbb{Z}_{2}^{n}$ to $\mathbb{F}$ is to index the family by two polynomials in $\mathbb{F}$. To pick a random element of the family, one randomly picks $a_{1}, a_{2} \in \mathbb{F}$ and defines $h(r)=a_{1} r+a_{2}$, where $r$ is treated like a polynomial in $\mathbb{F}$ and multiplication and addition is performed over $\mathbb{F} 6$ The final secret key is a random polynomial $s \in \mathbb{F}$. Thus the secret keys are $\left(S, a_{1}, a_{2}, s\right)$.

In the protocol, the Tag chooses an $r \in \mathbb{Z}_{2}^{n}$ and sends it to the Reader, who replies with a randomly-chosen $c \in \mathbb{F}$. The Tag receives the $c$ computes $f^{\chi}(r)=$ $S r+e \in \mathbb{Z}_{2}^{m}$, and treats the result as a polynomial in $\mathbb{F}$. He then multiplies it by $s$ and adds it to $h(r) c=\left(a_{1} r+a_{2}\right) c$, and sends the resulting $z=f^{\chi}(r) s+h(r) c$

\footnotetext{
${ }^{6}$ To be able to treat $r$ as an element of $\mathbb{F}$, it is important that $m \geq n$. If $m<n$, then one can define the pairwise-independent function differently (e.g. $h(r)=a_{1} r_{1}+$ $\left.\ldots a_{k} r_{k}+a_{k+1}\right)$, where $\left.r=r_{1}|\cdots| r_{k}\right)$.
} 
to the Reader. The reader computes $f(r)=S r$ and $s^{-1}(z-h(r) c)$, and accepts if the weight of $f(r)-s^{-1}(z-h(r) c)$ is less than or equal to $\tau^{\prime}$.

Notice that the protocol would be exactly the same for the Toeplitz-LPN ${ }_{n, \tau}$ problem, with the only difference being how $S$ is defined. By having $S$ be a Toeplitz matrix, the key storage space shrinks from $m n+3 m$ to $n+4 m$, and the matrix-vector multiplication $S r$ can be computed more efficiently. The Ring$\mathrm{LPN}_{n, \tau}$ protocol would also work in essentially the same way. In this case, we set $m=n$ and have $\mathbb{D}=\mathbb{F}$. The secret key $S$ will just be a random polynomial in $\mathbb{F}$ just like $s, a_{1}$, and $a_{2}$. Thus $S r$ will simply be a multiplication of two polynomials in the field $\mathbb{F}$.

Lemma 3.1. The completeness of the authentication protocol is $\operatorname{Pr}_{e \sim \chi}\left[\|e\| \leq \tau^{\prime}\right]$. And in particular, if the weak-PRF is not randomized, the completeness is 1.

Proof. The Tag sets $z \leftarrow f^{\chi}(r) s+h(r) c=(f(r)+e) s+h(r) c$, where $e \sim \chi$. Thus $f(r)-s^{-1}(z-h(r) c)=e$, and so the Reader accepts whenever $\| f(r)-$ $s^{-1}(z-h(r) c)\|=\| e \| \leq \tau^{\prime}$.

\section{Security of the Authentication Scheme}

Theorem 4.1 Suppose that the authentication protocol in Figure 2 has completeness $\kappa$ and there is a man-in-the-middle adversary who successfully breaks this scheme with probability $\epsilon$ while making at most $q_{v}$ verification queries. Then there exists an algorithm which, in the same amount of time, has advantage $\frac{1}{2}\left(\kappa^{q_{v}-1}\left(\epsilon / q_{v}-1 /|\mathbb{F}|\right)^{2}-\beta\left(2 \tau^{\prime}\right) /|\mathbb{F}|\right)$ in breaking the (randomized) weak-PRF assumption of the family $\mathcal{F}$.

Proof. If an adversary making $q_{v}$ verification queries wins the game, then one of these $q_{v}$ queries can be thought of as the "winning query". By "winning query", we mean that it is the first accepted query such that $\left(r_{i}^{\prime}, c_{i}^{\prime}, z_{i}^{\prime}\right) \neq(0,0,0)$ (where $r_{i}^{\prime}, c_{i}^{\prime}, z_{i}^{\prime}$ are as in Figure 1). Once the Adversary sends such a query, he wins the game. If the Adversary has an $\epsilon$ success probability of winning the MIM-game, then by an averaging argument there must be some integer $i^{*} \leq q_{v}$ such that the probability that the Adversary wins the game and query number $i^{*}$ is the "winning query" is at least $\epsilon / q_{v}$. For the rest of the proof, we will assume that we know this $i^{*}$ (which can be determined a priori by running the adversary on known inputs.)

The Challenger gives us ordered pairs $\left(r_{i}, y_{i}\right) \in \mathbb{D} \times \mathbb{F}$ where the $r_{i}$ are uniformly random in $\mathbb{D}$ and the $y_{i}$ are either uniformly random in $\mathbb{F}$ or equal to $f^{\chi}\left(r_{i}\right)$ (where $f$ is a randomly-chosen function from the (randomized) weak-PRF family $\mathcal{F}$ with noise $\chi$ ). We will show how to use the adversary who breaks the authentication protocol with the $i^{*}$ th winning query to decide which of the two distribution the Challenger is outputting.

Our security proof is most naturally divided into two cases. In the first case, the adversary does not modify the $r_{i^{*}}$, in other words, $\left(r_{i^{*}}^{\prime}, c_{i^{*}}^{\prime}, z_{i^{*}}^{\prime}\right)=\left(0, c_{i^{*}}^{\prime}, z_{i^{*}}^{\prime}\right)$. In the second case, the $r_{i^{*}}$ is modified in the winning query. The manner in which 


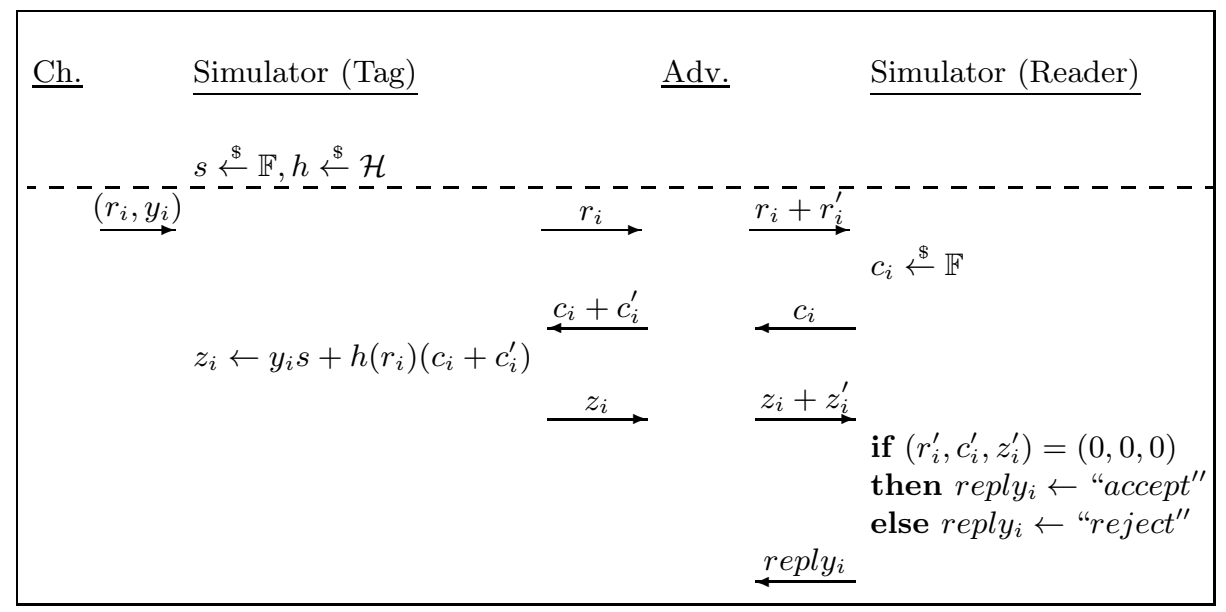

Fig. 3. Simulating the Tag and the Reader before the Adversary's $i^{*}$ th verification query. If the weak-PRF is not randomized, then we set the secret key $\mathrm{s}=1$ instead of choosing it at random from $\mathbb{F}$.

the simulator uses the Adversary's winning query to respond to the challenger differs based on whether $r_{i^{*}}^{\prime}$ is 0 or not. For the purposes of improved readability, throughout the rest of the paper, we will drop the subscript $i^{*}$ from all variables in the winning query. So for example, instead of writing $r_{i^{*}}^{\prime}$, we simply write $r^{\prime}$.

Answering the Challenger when $\boldsymbol{r}^{\prime}=\mathbf{0}$. We first show how to simulate the Tag and the Reader before the Adversary's $i^{*}$ th verification query (see Figure 3). We pick a random $s \in \mathbb{F}$ and $h \in \mathcal{H}$ as the secret keys, and upon receiving a pair $\left(r_{i}, y_{i}\right)$ from the Challenger, we send $r_{i}$ to the Adversary. The Adversary can then modify this and forward $r_{i}+r_{i}^{\prime}$ to the Reader. The Reader picks a random $c_{i} \in \mathbb{F}$, sends it to the adversary, who then sends the possibly modified challenge $c_{i}+c_{i}^{\prime}$ to the Tag. The Simulator playing as the Tag computes $h\left(r_{i}\right)\left(c_{i}+c_{i}^{\prime}\right)$ using his secret key $h$, and then uses the $y_{i}$ received from the challenger together with his other secret key $s$, to send $z_{i}=y_{i} s+h\left(r_{i}\right)\left(c_{i}+c_{i}^{\prime}\right)$. After receiving $z_{i}$, the Adversary may send $z_{i}+z_{i}^{\prime}$ to the verifier and make a verification query.

Notice that if the Challenger sends $\left(r_{i}, y_{i}=f^{\chi}\left(r_{i}\right)\right)$, then the responses of the Tag are exactly what they should be if the secret key were $(f, s, h)$. Thus if $\left(r_{i}^{\prime}, c_{i}^{\prime}, z_{i}^{\prime}\right)=(0,0,0)$, the Reader who always sends "accept" is correct with probability $\kappa$ (the completeness of the protocol). And if $\left(r_{i}^{\prime}, c_{i}^{\prime}, z_{i}^{\prime}\right) \neq(0,0,0)$, the response of "reject" is also correct since the $i^{*}$ th verification query has not yet been reached. Because the simulator has faithfully simulated the valid Tag and Reader up to this point with probability $\kappa^{q_{v}-1}$, the Adversary's $i^{*}$ th query will be the "winning one" (i.e. $\left(r^{\prime}, c^{\prime}, z^{\prime}\right) \neq(0,0,0)$ and $\| f\left(r+r^{\prime}\right)-$ $\left.s^{-1}\left(\left(z+z^{\prime}\right)-h\left(r+r^{\prime}\right) c\right) \| \leq \tau^{\prime}\right)$ with probability $\kappa^{q_{v}-1} \epsilon / q_{v}$. Additionally, because $z=f^{\chi}(r) s+h(r)\left(c+c^{\prime}\right)$, we also have $\left\|f(r)-s^{-1}\left(z-h(r)\left(c+c^{\prime}\right)\right)\right\| \leq \tau^{\prime}$. 


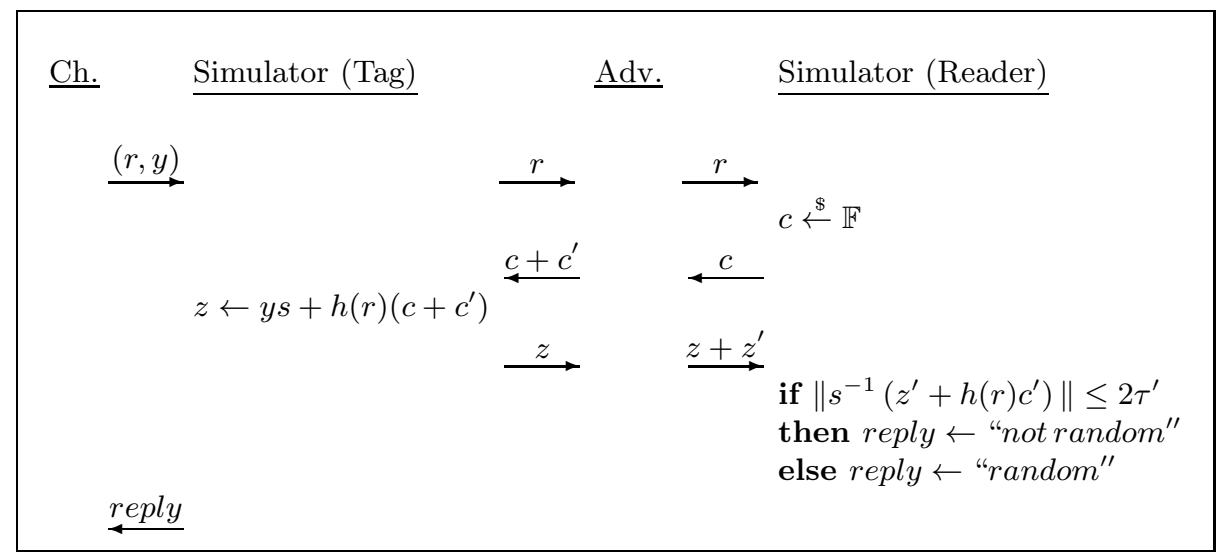

Fig. 4. Answering the Challenger after the Adversary's $i^{*}$ th verification query if $r^{\prime}=0$

Thus by the triangular inequality, we obtain $\left\|s^{-1}\left(z^{\prime}+h(r) c^{\prime}\right)\right\| \leq 2 \tau^{\prime}$. If this condition is satisfied, we respond to the challenger that the ordered pairs he was sending were indeed of the form $\left(r_{i}, y_{i}=f^{\chi}\left(r_{i}\right)\right)$.

Lemma 4.1. If the challenger were sending valid pairs, i.e. $\left(r_{i}, y_{i}=f \chi\left(r_{i}\right)\right)$ $\forall i$, then $\left\|s^{-1}\left(z^{\prime}+h(r) c^{\prime}\right)\right\| \leq 2 \tau^{\prime}$ should be satisfied with probability at least $\kappa^{q_{v}-1} \epsilon / q_{v}$.

On the other hand, if the Challenger were sending uniformly random pairs $\left(r_{i}, y_{i}\right) \in(\mathbb{D}, \mathbb{F})$ then we will show that the adversary is not be able (except with a negligible probability) to come up with $\left(c^{\prime}, z^{\prime}\right) \neq(0,0)$ that satisfy the inequality $\left\|s^{-1}\left(z^{\prime}+h(r) c^{\prime}\right)\right\| \leq 2 \tau^{\prime}$. Notice that if the $y_{i}$ are uniform and independent of the $r_{i}$, the secret keys $h, s$ chosen by the simulator are information-theoretically hidden throughout the interaction in Figure 3. Therefore the Adversary's behavior will be exactly the same as in the case where $s$ and $h$ are chosen after he sends his $i^{*}$ th query. In Lemma 4.2, we use this to show that even an all-powerful adversary cannot produce a query $z+z^{\prime}$ such that $\left\|s^{-1}\left(z^{\prime}+h(r) c^{\prime}\right)\right\| \leq 2 \tau^{\prime}$, except with probability $\beta\left(2 \tau^{\prime}\right) /|\mathbb{F}|$.

Lemma 4.2. If the ordered pairs $\left(r_{i}, y_{i}\right)$ sent by the challenger are uniformly random in $\mathbb{D} \times \mathbb{F}$, then the probability that even an all-powerful adversary can output $\left(c^{\prime}, z^{\prime}\right) \neq(0,0)$ such that $\left\|s^{-1}\left(z^{\prime}+h(r) c^{\prime}\right)\right\| \leq 2 \tau^{\prime}$ is at most $\left|\beta\left(2 \tau^{\prime}\right)\right| /|\mathbb{F}|$.

Proof. We first handle the case where $f$ is a weak-PRF without any noise (i.e. the support of the distribution $\chi$ is 0 and $\left.\tau^{\prime}=0\right)$. In this case, the extra random key $s$ is not necessary in the protocol (i.e. $s=1$ ) and so the condition $\left\|s^{-1}\left(z^{\prime}+h(r) c^{\prime}\right)\right\| \leq 2 \tau^{\prime}$ becomes $0=z^{\prime}+h(r) c^{\prime}$. Since $y$ is uniformly random in $\mathbb{F}$ and independent of everything else, the value $z$ that the adversary receives is also uniformly random and independent of the pairwise independent hash function $h$. Thus the adversary will behave in the same way if the function 
$h$ were chosen after the adversary chooses $c^{\prime}$ and $z^{\prime}$. Notice that the adversary must set $c^{\prime} \neq 0$ because otherwise $z^{\prime}$ is also necessarily 0 . Thus,

$$
\forall r \in \mathbb{D}, z^{\prime} \in \mathbb{F}, c^{\prime} \in \mathbb{F} \backslash\{0\}, \underset{h}{\operatorname{Pr}}\left[0=z^{\prime}+h(r) c^{\prime}\right]=\operatorname{Pr}_{h}\left[h(r)=-z^{\prime} c^{\prime-1}\right]=1 /|\mathbb{F}| .
$$

The proof for case where the support of $\chi$ is not restricted to 0 is similar, except that it also uses the unpredictability of the key $s$. The full proof is given in the full version.

Answering the Challenger When $\boldsymbol{r}^{\prime} \neq \mathbf{0}$. We now deal with the case where the Adversary's winning query changes the randomness $r$ to $r+r^{\prime}$. Performing the simulation until the $i^{*}$ th query is exactly the same as before (i.e. see Figure 31). Similarly, if the Challenger sends $\left(r_{i}, y_{i}=f^{\chi}\left(r_{i}\right)\right)$, then the responses of the Tag are exactly what they should be if the secret key were $(f, s, h)$. And so, as before, the Adversary's $i^{*}$ th query will be the "winning one" with probability $\kappa^{q_{v}-1} \epsilon / q_{v}$. The difference from the previous part lies in how we will use the Adversary's response in the $i^{*}$ th query to respond to the challenger. Unlike the previous case, we will now need to rewind the adversary and receive two responses for the same value of $r+r^{\prime}$ (see Figure 5). By the Reset Lemma 2, Lemma 3.1], the adversary will respond correctly to two distinct challenges $c_{0}$ and $c_{1}$ with probability $\kappa^{q_{v}-1}\left(\epsilon / q_{v}-1 /|\mathbb{F}|\right)^{2}$. If the Adversary successfully replies to the two queries, then we have $\left\|f\left(r+r^{\prime}\right)-s^{-1}\left(z_{0}+z_{0}^{\prime}-h\left(r+r^{\prime}\right) c_{0}\right)\right\| \leq \tau^{\prime}$ and $\left\|f\left(r+r^{\prime}\right)-s^{-1}\left(z_{1}+z_{1}^{\prime}-h\left(r+r^{\prime}\right) c_{1}\right)\right\| \leq \tau^{\prime}$. Thus, by the triangle inequality, we have the condition $\left\|s^{-1}\left(\left(z_{0}+z_{0}^{\prime}\right)-\left(z_{1}+z_{1}^{\prime}\right)-h\left(r+r^{\prime}\right)\left(c_{0}-c_{1}\right)\right)\right\| \leq 2 \tau^{\prime}$. If this is satisfied, we reply to the challenger that the ordered pairs he was sending were indeed of the form $\left(r_{i}, y_{i}=f^{\chi}\left(r_{i}\right)\right)$.

Lemma 4.3. If the Challenger were sending valid pairs, i.e. $\left(r_{i}, y_{i}=f^{\chi}\left(r_{i}\right)\right)$ $\forall i$, then $\left\|s^{-1}\left(\left(z_{0}+z_{0}^{\prime}\right)-\left(z_{1}+z_{1}^{\prime}\right)-h\left(r+r^{\prime}\right)\left(c_{0}-c_{1}\right)\right)\right\| \leq 2 \tau^{\prime}$ should be satisfied with probability at least $\kappa^{q_{v}-1}\left(\epsilon / q_{v}-1 /|\mathbb{F}|\right)^{2}$.

On the other hand, if the Challenger were sending uniformly random pairs $\left(r_{i}, y_{i}\right) \in(\mathbb{D}, \mathbb{F})$ then we will show that the adversary is not be able (except with a negligible probability) to come up with $\left(r^{\prime}, c^{\prime}, z^{\prime}\right)$ where $r^{\prime} \neq 0$ that satisfy $\left\|s^{-1}\left(\left(z_{0}+z_{0}^{\prime}\right)-\left(z_{1}+z_{1}^{\prime}\right)-h\left(r+r^{\prime}\right)\left(c_{0}-c_{1}\right)\right)\right\| \leq 2 \tau^{\prime}$. As before, notice that if the $y_{i}$ are uniform and independent of the $r_{i}$, the secret keys $h, s$ chosen by the simulator are information-theoretically hidden throughout the interaction in Figure 3. Therefore the Adversary's behavior will be exactly the same as in the case where $s$ and $h$ are chosen after he outputs his $i^{*}$ th query the first time. When we rewind the Adversary, we also end up rewinding the left-hand side of the simulator, which will end up revealing some information about $h$. But we use the pairwise-independent property of $h$ to show (in Lemma 4.4) that even an all-powerful adversary still cannot produce a query $z+z^{\prime}$ such that $\left\|s^{-1}\left(\left(z_{0}+z_{0}^{\prime}\right)-\left(z_{1}+z_{1}^{\prime}\right)-h\left(r+r^{\prime}\right)\left(c_{0}-c_{1}\right)\right)\right\| \leq 2 \tau^{\prime}$, except with probability $\beta\left(2 \tau^{\prime}\right) /|\mathbb{F}|$. 


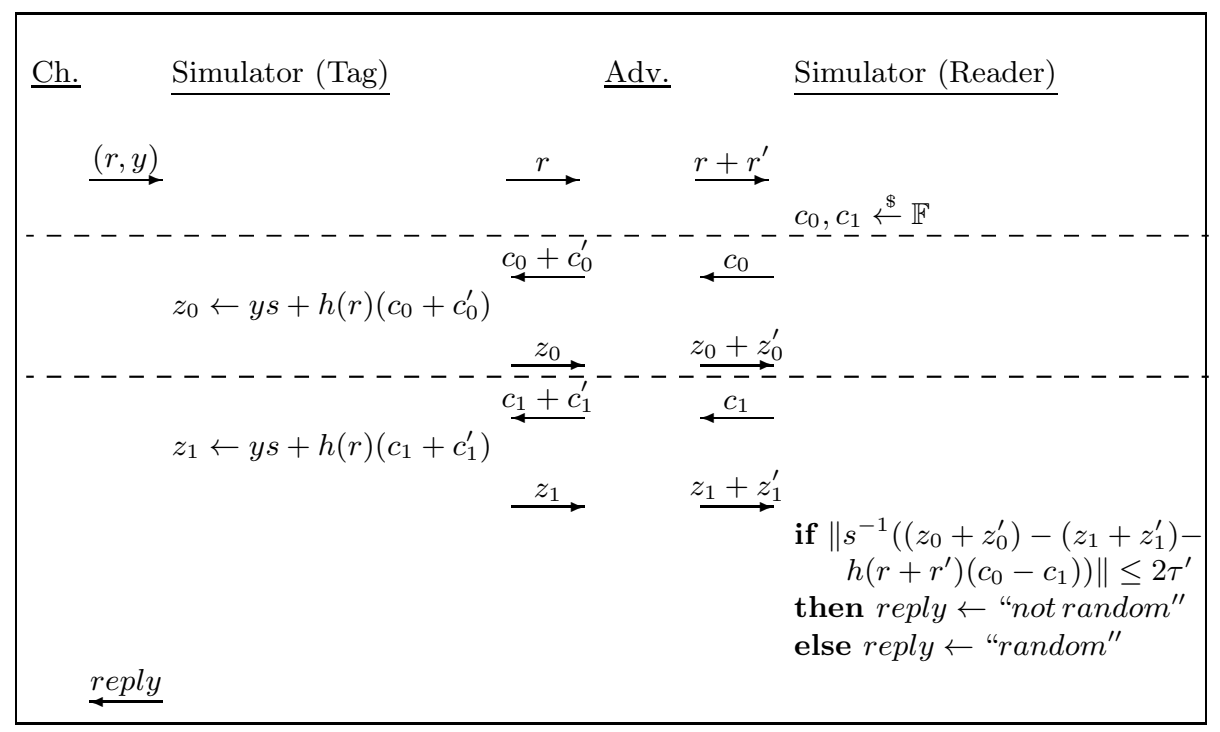

Fig. 5. Answering the Challenger after the Adversary's $i^{*}$ th verification query if $r^{\prime} \neq 0$

Lemma 4.4. If the ordered pairs $\left(r_{i}, y_{i}\right)$ sent by the challenger are uniformly random in $\mathbb{D} \times \mathbb{F}$, and $r^{\prime} \neq 0$, and $c_{0} \neq c_{1}$, then the probability that even an all-powerful adversary can output $z_{0}^{\prime}$ and $z_{1}^{\prime}$ such that $\| s^{-1}\left(\left(z_{0}+z_{0}^{\prime}\right)-\left(z_{1}+\right.\right.$ $\left.\left.z_{1}^{\prime}\right)-h\left(r+r^{\prime}\right)\left(c_{0}-c_{1}\right)\right) \| \leq 2 \tau^{\prime}$ is at most $\left|\beta\left(2 \tau^{\prime}\right)\right| /|\mathbb{F}|$.

Proof. For simplicity, we will define $w=\left(z_{0}+z_{0}^{\prime}\right)-\left(z_{1}+z_{1}^{\prime}\right)$. The information given to the adversary (in the two rewindings) by the simulator playing as the tag is $z_{0}=y s+h(r)\left(c_{0}+c_{0}^{\prime}\right)$ and $z_{1}=y s+h(r)\left(c_{1}+c_{1}^{\prime}\right)$. This is exactly the same as receiving $z_{0}$ and $\tilde{z}=z_{0}-z_{1}=h(r)\left(c_{0}+c_{0}^{\prime}-\left(c_{1}+c_{1}^{\prime}\right)\right)$. Notice that since $z_{0}$ contains the term $y s$, the value of $z_{0}$ is uniform and independent of the function $h$. The value of $\tilde{z}$, on the other hand, does depend on $h(r)$. So the behavior of the adversary would be unchanged if we chose $z_{0}$ uniformly at random, chose a random element $u$ for $h(r)$ and set $\tilde{z}=h(r)\left(c_{0}+c_{0}^{\prime}-\left(c_{1}+c_{1}^{\prime}\right)\right)$, and then after the adversary picks $z_{0}^{\prime}, z_{1}^{\prime}$, we finally choose $h$ (conditioned on the already set value of $h(r))$. Thus we have that $\forall t \in \beta\left(2 \tau^{\prime}\right), c_{0} \neq c_{1} \in \mathbb{F}, r \in \mathbb{D}, r^{\prime} \neq 0, w, s, u \in \mathbb{F}$

$$
\begin{aligned}
\operatorname{Pr}_{h}\left[s^{-1}\left(w-h\left(r+r^{\prime}\right)\left(c_{0}-c_{1}\right)\right)\right. & =t \mid h(r)=u]=\underset{h}{\operatorname{Pr}}\left[h\left(r+r^{\prime}\right)\right. \\
& \left.=(w-s t)\left(c_{0}-c_{1}\right)^{-1} \mid h(r)=u\right]=1 /|\mathbb{F}|
\end{aligned}
$$

where $\left(c_{0}-c_{1}\right)^{-1}$ exists since we assumed $c_{0} \neq c_{1}$ and the last equality is true because $h$ is a pairwise-independent function and $r^{\prime} \neq 0$.

Combining Lemmas 4.1, 4.2, 4.3, and 4.4 gives the statement of Theorem 4.1. 


\section{Discussion and Open Problems}

In this work we presented a very efficient 3-round authentication scheme that utilizes only one call to a (randomized) weak-PRF, and proved it secure against sequential MIM attacks. The security proof can be extended to the scenario where Adversary is allowed to concurrently interact with many copies of the Reader (this will be shown in the full version of the paper). Another simple extension is the conversion of the scheme into an interactive message authentication scheme by sending $r \| \mu$ in lieu of just $r$ in the first round and setting $z \leftarrow f^{\chi}(r) s+h(r \| \mu) c$ in the third round, where $\mu$ is the message digest.

We believe that the most interesting (theoretical) question left open by our work is to construct a MIM-secure authentication scheme that is secure in the full concurrent setting (i.e. where the Adversary is also allowed to concurrently interact with multiple provers). Such a scheme can be easily constructed by first creating a PRF from a weak-PRF using $O(n)$ calls to the weak-PRF. The challenge is thus to construct such a scheme using just one, or even a constant number, of (randomized) weak-PRF invocations.

Acknowledgements. We are very grateful to Eike Kiltz and Krzysztof Pietrzak for numerous discussions pertaining to their work on the LPN problem. We also thank Daniel Wichs and the other anonymous CRYPTO 2013 reviewers for their valuable comments - in particular mentioning the application to interactive MACs.

\section{References}

1. Banerjee, A., Peikert, C., Rosen, A.: Pseudorandom functions and lattices. In: Pointcheval, D., Johansson, T. (eds.) EUROCRYPT 2012. LNCS, vol. 7237, pp. 719-737. Springer, Heidelberg (2012)

2. Bellare, M., Palacio, A.: Gq and schnorr identification schemes: Proofs of security against impersonation under active and concurrent attacks. In: Yung, M. (ed.) CRYPTO 2002. LNCS, vol. 2442, pp. 162-177. Springer, Heidelberg (2002)

3. Blum, A., Furst, M., Kearns, M., Lipton, R.J.: Cryptographic primitives based on hard learning problems. In: Stinson, D.R. (ed.) CRYPTO 1993. LNCS, vol. CRYPTO, pp. 278-291. Springer, Heidelberg (1994)

4. Bogdanov, A., Knudsen, L.R., Leander, G., Paar, C., Poschmann, A., Robshaw, M., Seurin, Y., Vikkelsoe, C.: Present: An ultra-lightweight block cipher. In: Paillier, P., Verbauwhede, I. (eds.) CHES 2007. LNCS, vol. 4727, pp. 450-466. Springer, Heidelberg (2007)

5. Damgård, I.B., Nielsen, J.B.: Expanding pseudorandom functions; or: From knownplaintext security to chosen-plaintext security. In: Yung, M. (ed.) CRYPTO 2002. LNCS, vol. 2442, pp. 449-464. Springer, Heidelberg (2002)

6. Dodis, Y., Kiltz, E., Pietrzak, K., Wichs, D.: Message authentication, revisited. In: Pointcheval, D., Johansson, T. (eds.) EUROCRYPT 2012. LNCS, vol. 7237, pp. 355-374. Springer, Heidelberg (2012)

7. Gilbert, H., Robshaw, M., Sibert, H.: An active attack against $\mathrm{HB}^{+}$- a provably secure lightweight authentication protocol. Cryptology ePrint Archive, Report $2005 / 237$ (2005) 
8. Gilbert, H., Robshaw, M.J.B., Seurin, Y.: Good variants of $\mathrm{HB}^{+}$are hard to find. In: Tsudik, G. (ed.) FC 2008. LNCS, vol. 5143, pp. 156-170. Springer, Heidelberg (2008)

9. Gilbert, H., Robshaw, M.J.B., Seurin, Y.: HB\# : Increasing the security and efficiency of $\mathrm{HB}^{+}$. In: Smart, N.P. (ed.) EUROCRYPT 2008. LNCS, vol. 4965, pp. 361-378. Springer, Heidelberg (2008)

10. Goldreich, O., Goldwasser, S., Micali, S.: How to construct random functions. J. ACM 33(4), 792-807 (1986)

11. Goldreich, O., Krawczyk, H., Luby, M.: On the existence of pseudorandom generators. SIAM J. Comput. 22(6), 1163-1175 (1993)

12. Guillou, L.C., Quisquater, J.-J.: A "Paradoxical" identity-based signature scheme resulting from zero-knowledge. In: Goldwasser, S. (ed.) CRYPTO 1988. LNCS, vol. 403, pp. 216-231. Springer, Heidelberg (1990)

13. Heyse, S., Kiltz, E., Lyubashevsky, V., Paar, C., Pietrzak, K.: Lapin: An efficient authentication protocol based on ring-lpn. In: Canteaut, A. (ed.) FSE 2012. LNCS, vol. 7549, pp. 346-365. Springer, Heidelberg (2012)

14. Hopper, N.J., Blum, M.: Secure human identification protocols. In: Boyd, C. (ed.) ASIACRYPT 2001. LNCS, vol. 2248, pp. 52-66. Springer, Heidelberg (2001)

15. Juels, A., Weis, S.A.: Authenticating pervasive devices with human protocols. In: Shoup, V. (ed.) CRYPTO 2005. LNCS, vol. 3621, pp. 293-308. Springer, Heidelberg (2005)

16. Katz, J., Shin, J.S.: Parallel and concurrent security of the $\mathrm{HB}$ and $\mathrm{HB}^{+}$protocols. In: Vaudenay, S. (ed.) EUROCRYPT 2006. LNCS, vol. 4004, pp. 73-87. Springer, Heidelberg (2006)

17. Katz, J., Shin, J.S., Smith, A.: Parallel and concurrent security of the HB and $\mathrm{HB}^{+}$protocols. J. Cryptology 23(3), 402-421 (2010)

18. Kiltz, E., Pietrzak, K., Cash, D., Jain, A., Venturi, D.: Efficient authentication from hard learning problems. In: Paterson, K.G. (ed.) EUROCRYPT 2011. LNCS, vol. 6632, pp. 7-26. Springer, Heidelberg (2011)

19. Leander, G., Paar, C., Poschmann, A., Schramm, K.: New lightweight des variants. In: Biryukov, A. (ed.) FSE 2007. LNCS, vol. 4593, pp. 196-210. Springer, Heidelberg (2007)

20. Lyubashevsky, V., Peikert, C., Regev, O.: On ideal lattices and learning with errors over rings. In: Gilbert, H. (ed.) EUROCRYPT 2010. LNCS, vol. 6110, pp. 1-23. Springer, Heidelberg (2010)

21. Maurer, U.M., Sjödin, J.: A fast and key-efficient reduction of chosen-ciphertext to known-plaintext security. In: Naor, M. (ed.) EUROCRYPT 2007. LNCS, vol. 4515, pp. 498-516. Springer, Heidelberg (2007)

22. Naor, M., Reingold, O.: Number-theoretic constructions of efficient pseudo-random functions. In: FOCS, pp. 458-467 (1997)

23. Naor, M., Reingold, O., Rosen, A.: Pseudorandom functions and factoring. SIAM J. Comput. 31(5), 1383-1404 (2002)

24. Ouafi, K., Overbeck, R., Vaudenay, S.: On the security of HB ${ }^{\#}$ against a manin-the-middle attack. In: Pieprzyk, J. (ed.) ASIACRYPT 2008. LNCS, vol. 5350, pp. 108-124. Springer, Heidelberg (2008)

25. Pietrzak, K.: A leakage-resilient mode of operation. In: Joux, A. (ed.) EUROCRYPT 2009. LNCS, vol. 5479, pp. 462-482. Springer, Heidelberg (2009)

26. Regev, O.: On lattices, learning with errors, random linear codes, and cryptography. J. ACM 56(6) (2009)

27. Schnorr, C.-P.: Efficient signature generation by smart cards. J. Cryptology 4(3), 161-174 (1991) 